\title{
SaltWalks: Vancouver, Nanaimo, Toronto
}

Randy Lee Cutler,

Emily Carr University, Vancouver, Canada 
SaltWalks (2013-ongoing) is a performance series that takes participants on site-specific salt tasting walks through different city neighborhoods. Each walk begins at a designated meeting point where fellow walkers sample five different salts, including table salt, Celtic sea salt, Himalayan rock salt, Hawaiian clay salt, and Alderwood smoked salt. These tastings are served from a custom salt apron designed specifically for walking. Our savory comparisons initiate far-ranging conversations exploring the uses of salt across time and different cultures. Through an engagement with this elemental mineral, these walks become a pedagogical platform that embodies aesthetic and philosophical enquiries into the importance of this substance to ritual, survival, health, industry and the imagination.

As the tasting samples move through participants' digestive systems, we wind our way through the public spaces of a given location. This transit through urban geographywhether streets, parks or shops-becomes the backdrop to explore the relationship that human civilizations have had with salt, from its importance to life and manufacturing, to its more aesthetic and political implications. An important historical link between salt and walking is Gandhi's 240-mile Salt March in 1930, also known as the Salt Satyagraha, where the activist and his followers protested the British salt monopoly and taxation system. The Salt March eventually led to the Indian independence movement. A discussion of the political role of walking in this context sets the stage for an embodied engagement with public assembly and materiality.

As the many permutations of salt's diverse implications unfold, participants explore the connections that salt has to food and memory through silent walking and personal reflection, as well as shared stories that reveal a range of culturally specific recollections. More broadly, this crystalized mineral is probed for its omnipresence in daily life, from modern plastics, cleaning agents, ceramics and leather, to cosmetics and pharmaceuticals to name but a few industrial applications. As we walk through a neighborhood, the ubiquity of salt is made manifest with references to the built environment such as concrete, glass and dyes as well as the products on offer in shop windows.

Where possible, shopkeepers are organized ahead of time to meet with the group and convey their expertise. For example, in the first iteration of SaltWalks (2013) commissioned by Access Gallery in Vancouver's Chinatown, I researched the mineral's cultural history and sought out local shop-keepers and guests to share their knowledge of the diverse aspects of sodium chloride. The resulting three thematic walks - herbal medicine, food preservation, and crystal structures - reflected something of the specific character of these neighborhoods.

SaltWalks highlights how walking can be understood as a form of mobile thinking. Springgay and Truman (2018) describe this as an entangled, transmaterial, affective practice of experimentation, where materiality and movement elicits emergent connections in each participant. It is also a form of what Donna Haraway (2015) calls a sympoesis where we walk and talk together, making meaning through new associations. Part knowledge sharing, part performance art, part community building exercise, participants engage in peripatetic discussions of the scientific, medicinal, anthropological and historical relevance of this ubiquitous mineral. 


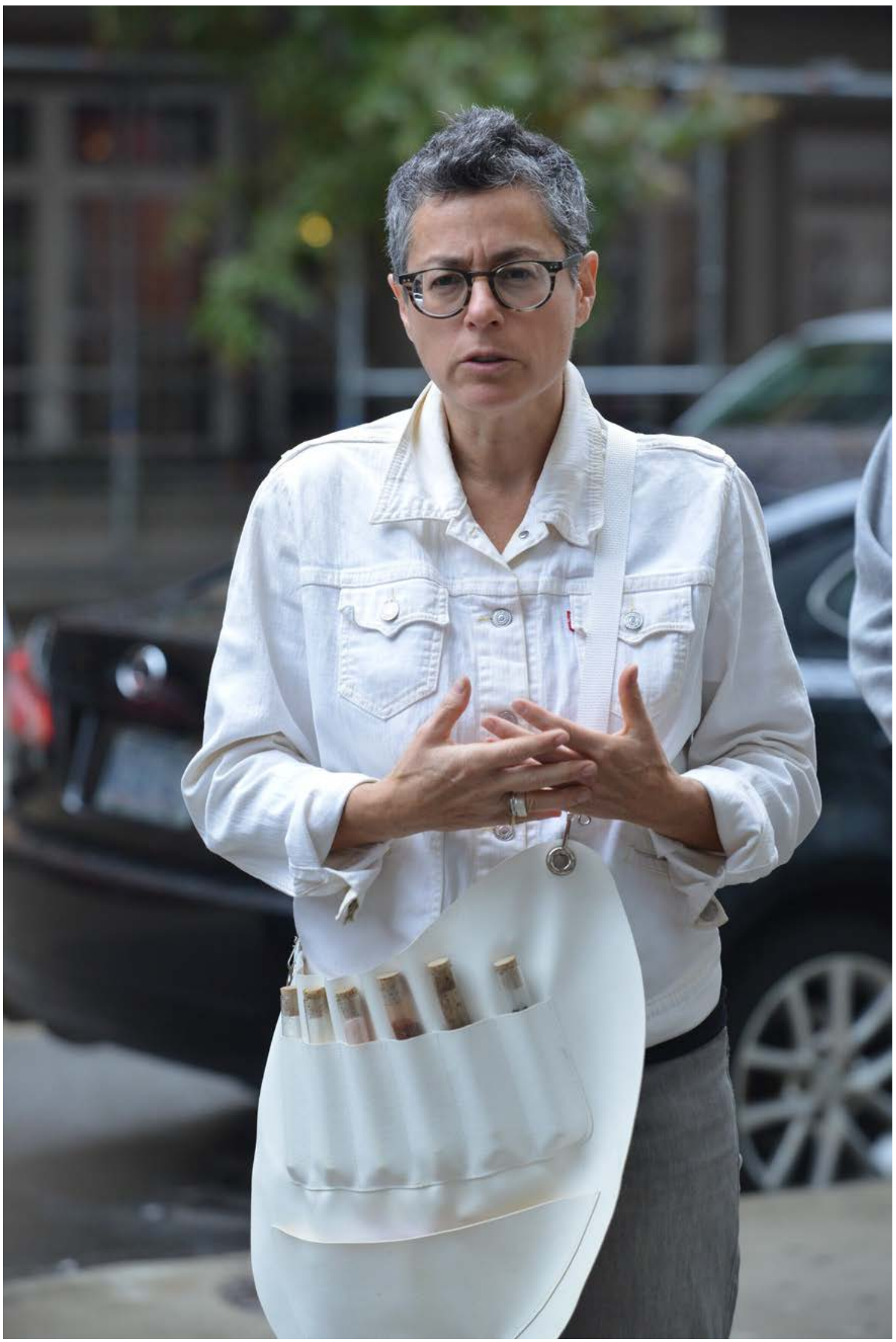

Figure 1: Randy Lee Cutler, 2016, SaltWalks: Toronto, 7a*11d International Festival of Performance Art, Toronto, Ontario. Photo credit: Henry Chan. 


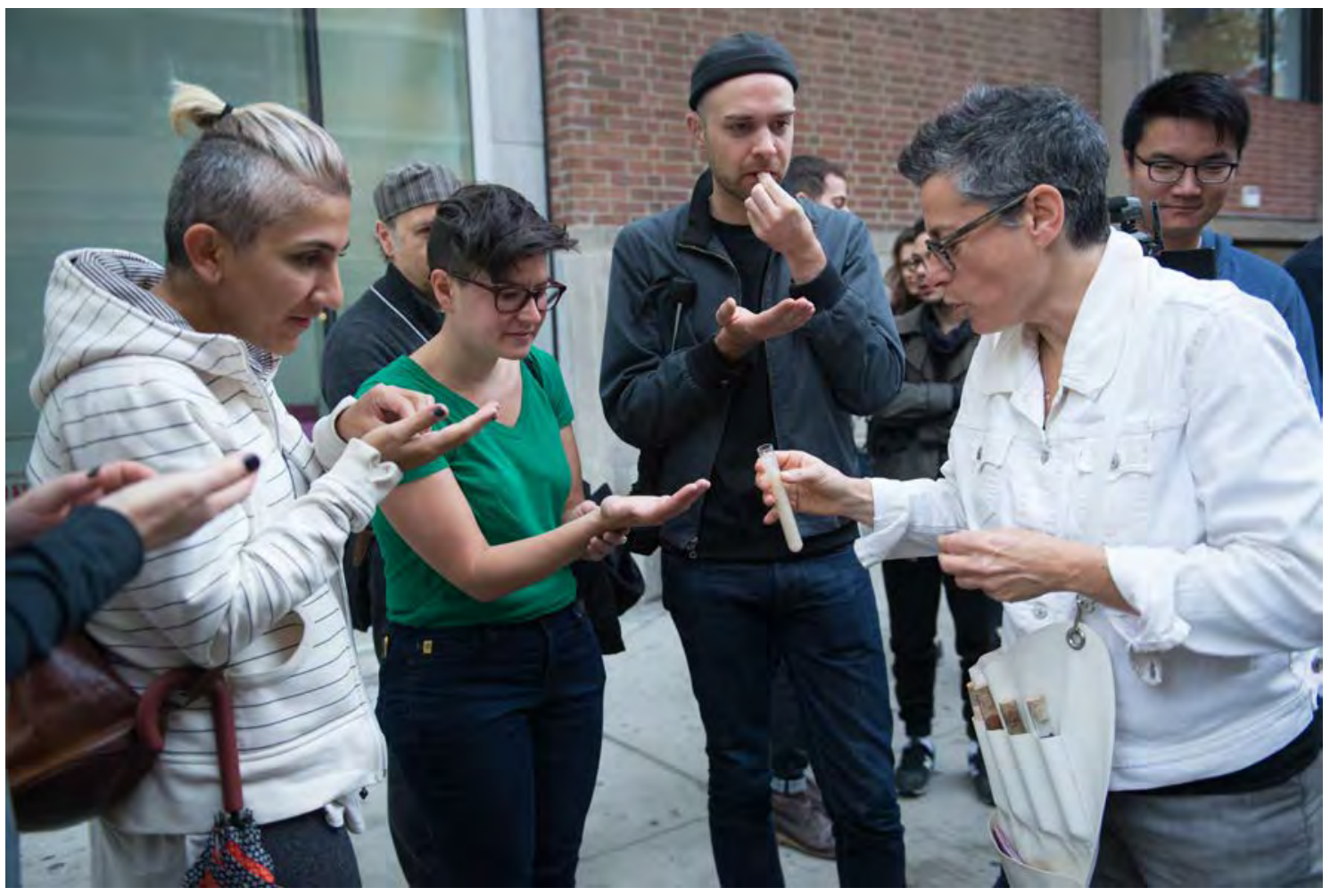

Figure 2: Randy Lee Cutler, 2016, SaltWalks: Toronto, 7a*11d International Festival of Performance Art, Toronto, Ontario, photo credit: Henry Chan.

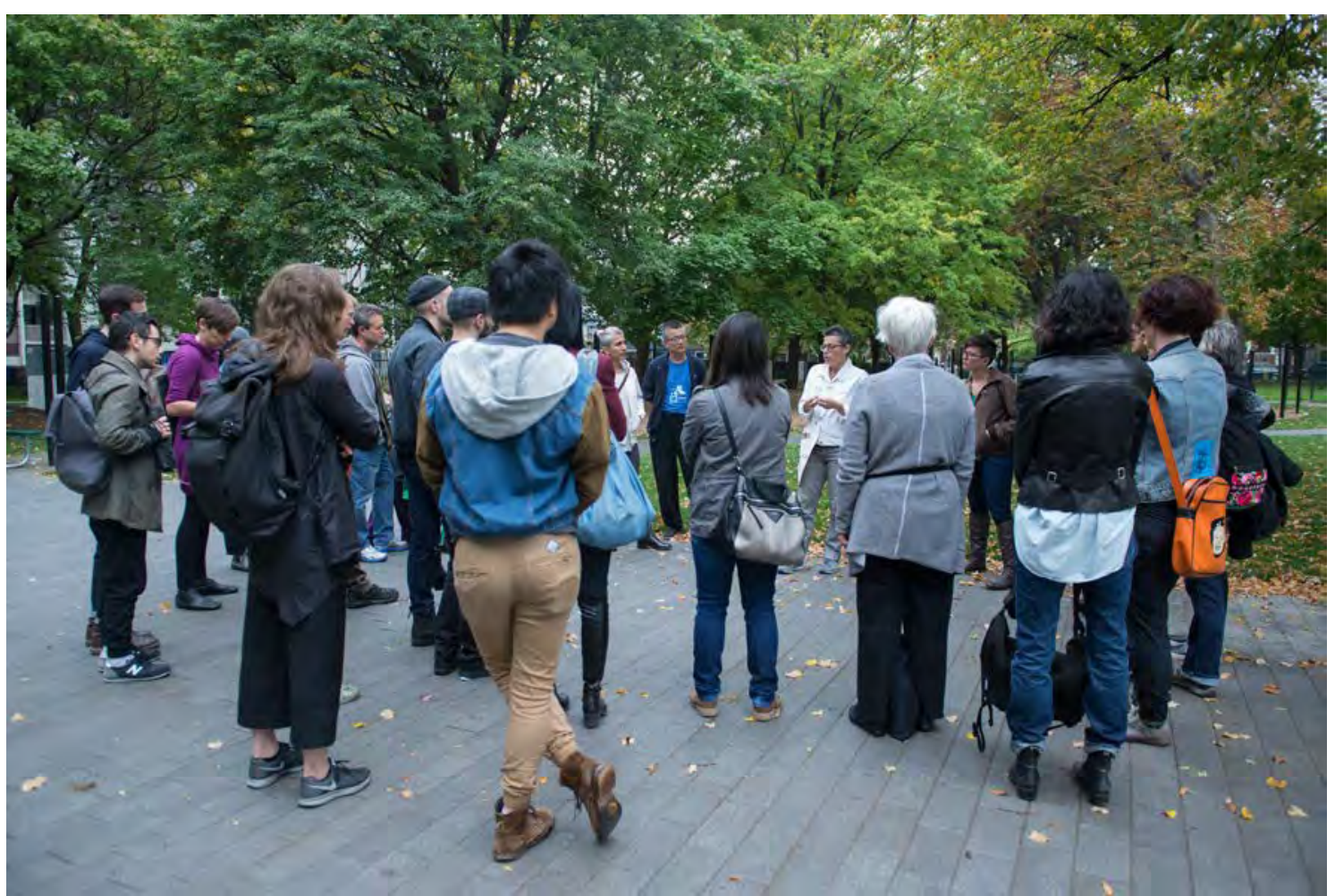

Figure 3: Randy Lee Cutler, 2016, SaltWalks: Toronto, 7a*11d International Festival of Performance Art, Toronto, Ontario. Photo credit: Henry Chan. 


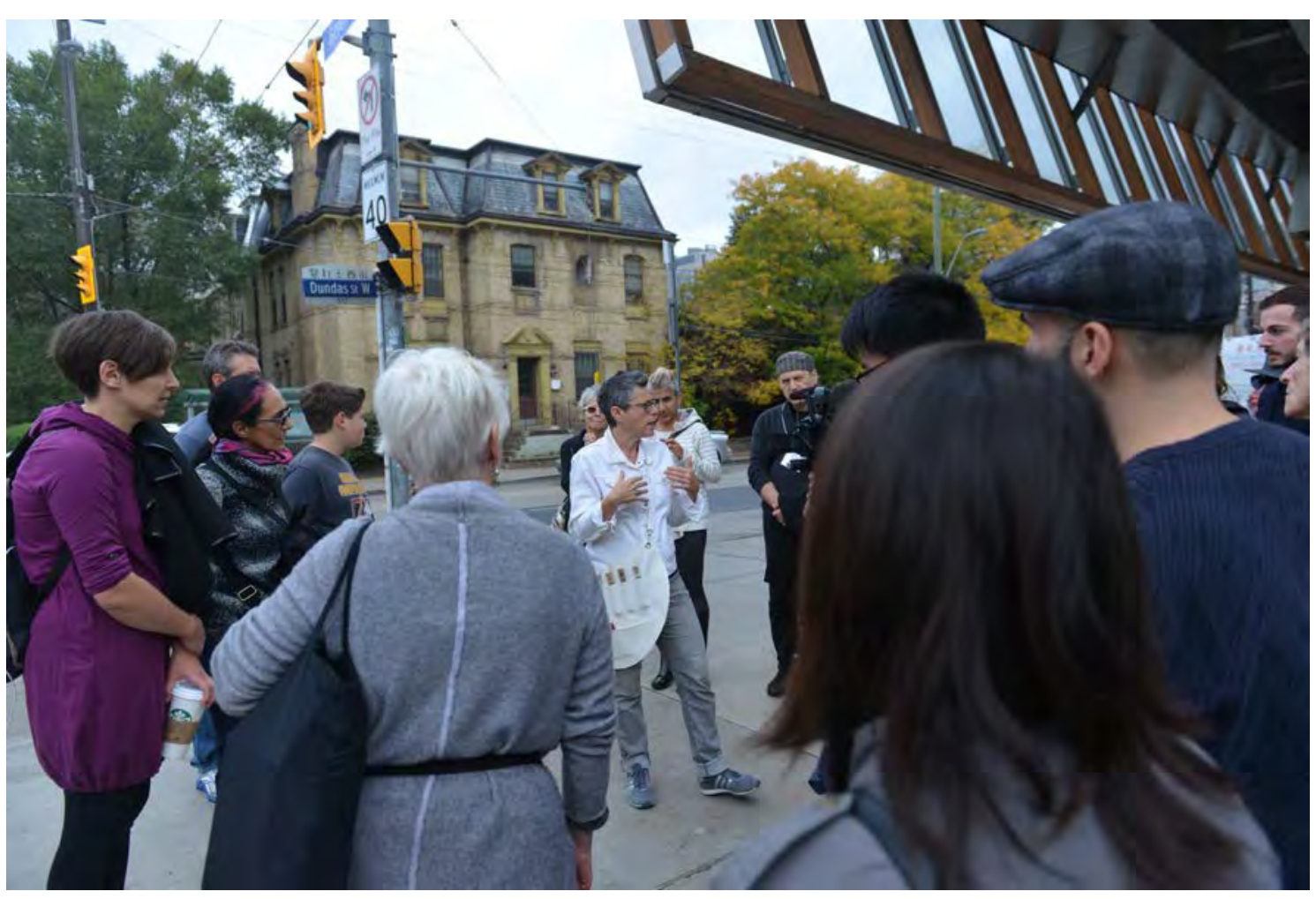

Figure 4: Randy Lee Cutler, 2016, SaltWalks: Toronto, 7a*11d International Festival of Performance Art, Toronto, Ontario. Photo credit: Henry Chan.

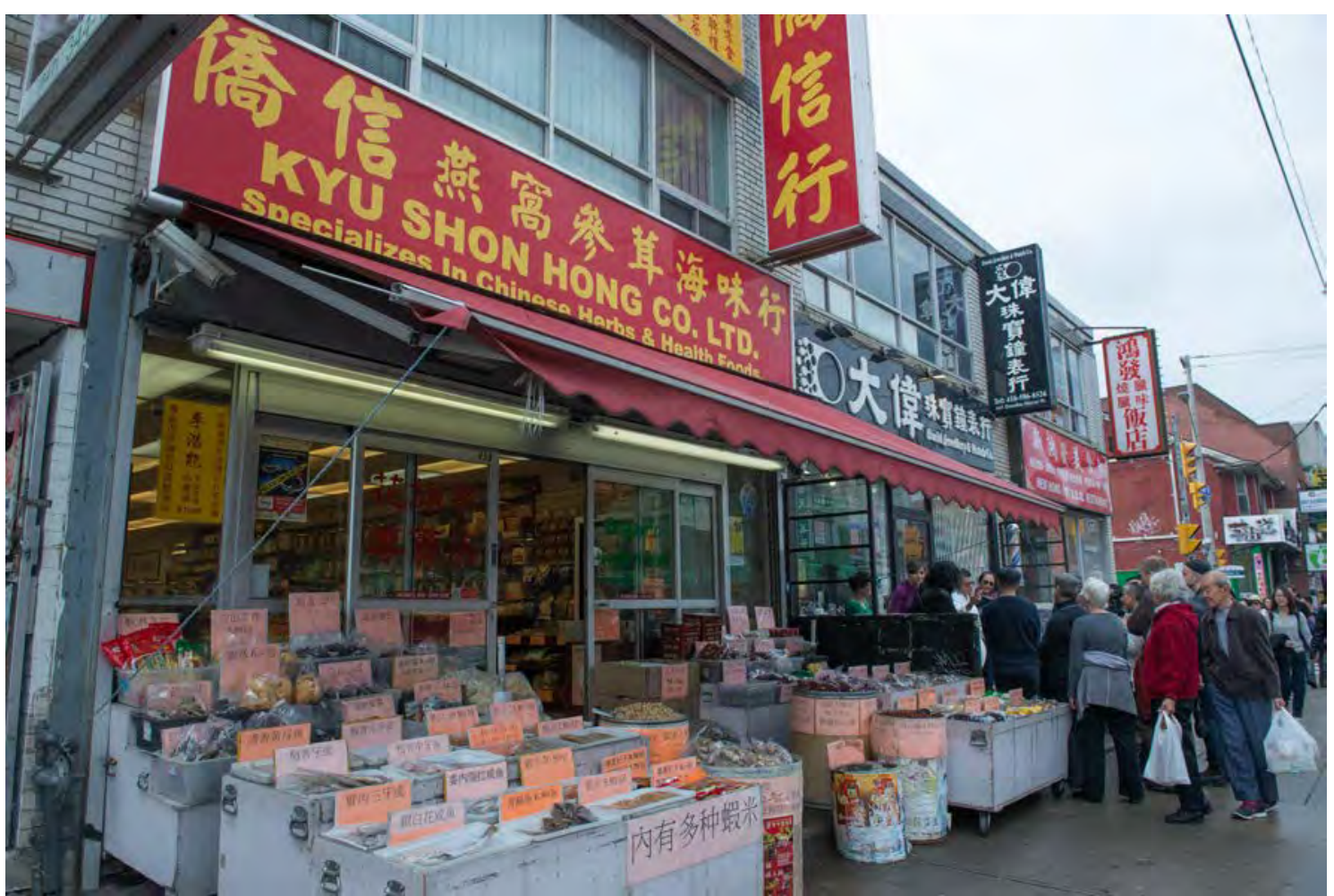

Figure 5: Randy Lee Cutler, 2016, SaltWalks: Toronto, 7a*11d International Festival of Performance Art, Toronto, Ontario. Photo credit: Henry Chan. 


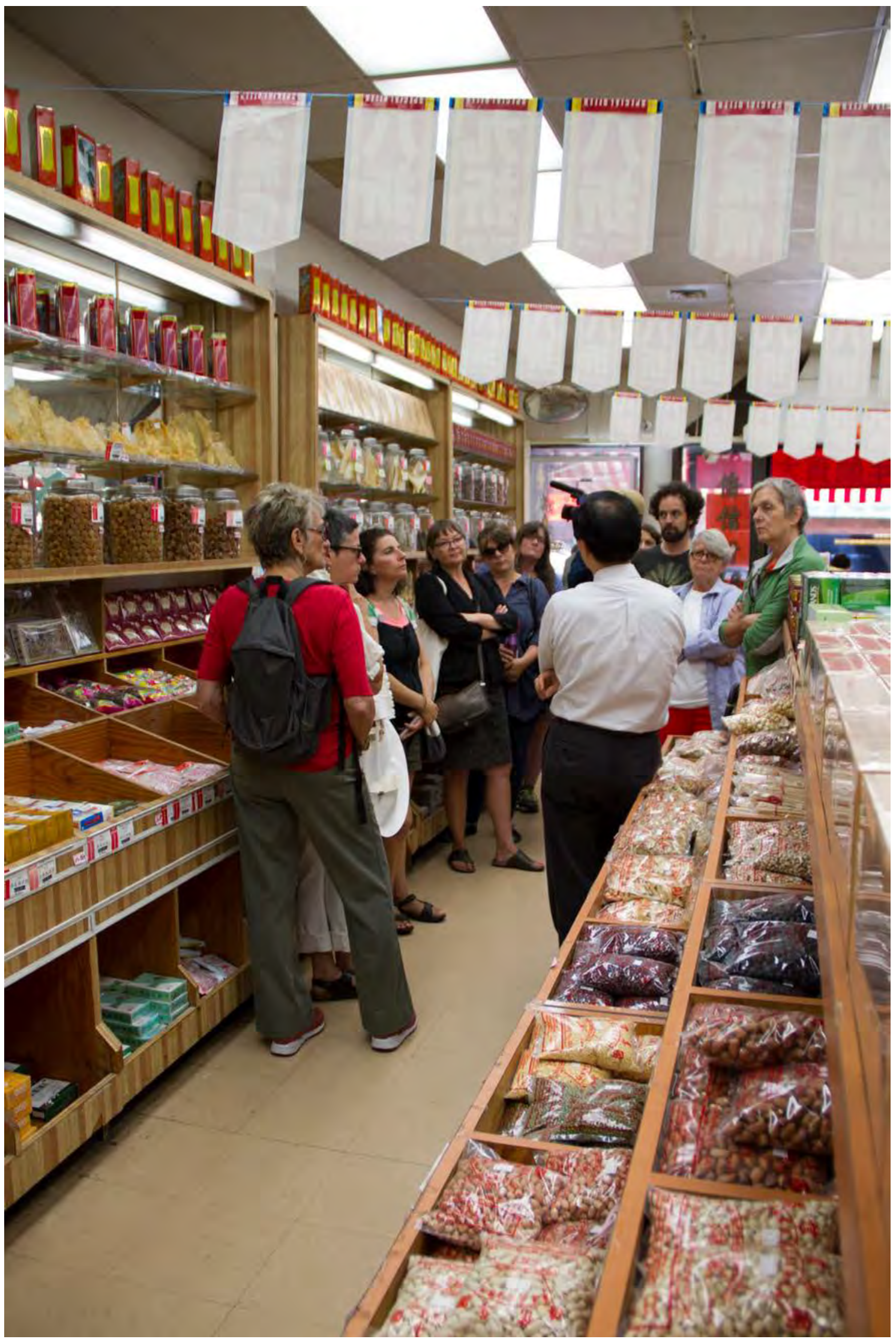

Figure 6: Randy Lee Cutler, 2013, SaltWalks: Vancouver, Access Art Gallery, Vancouver, BC. Photo credit: Dennis Ha. 


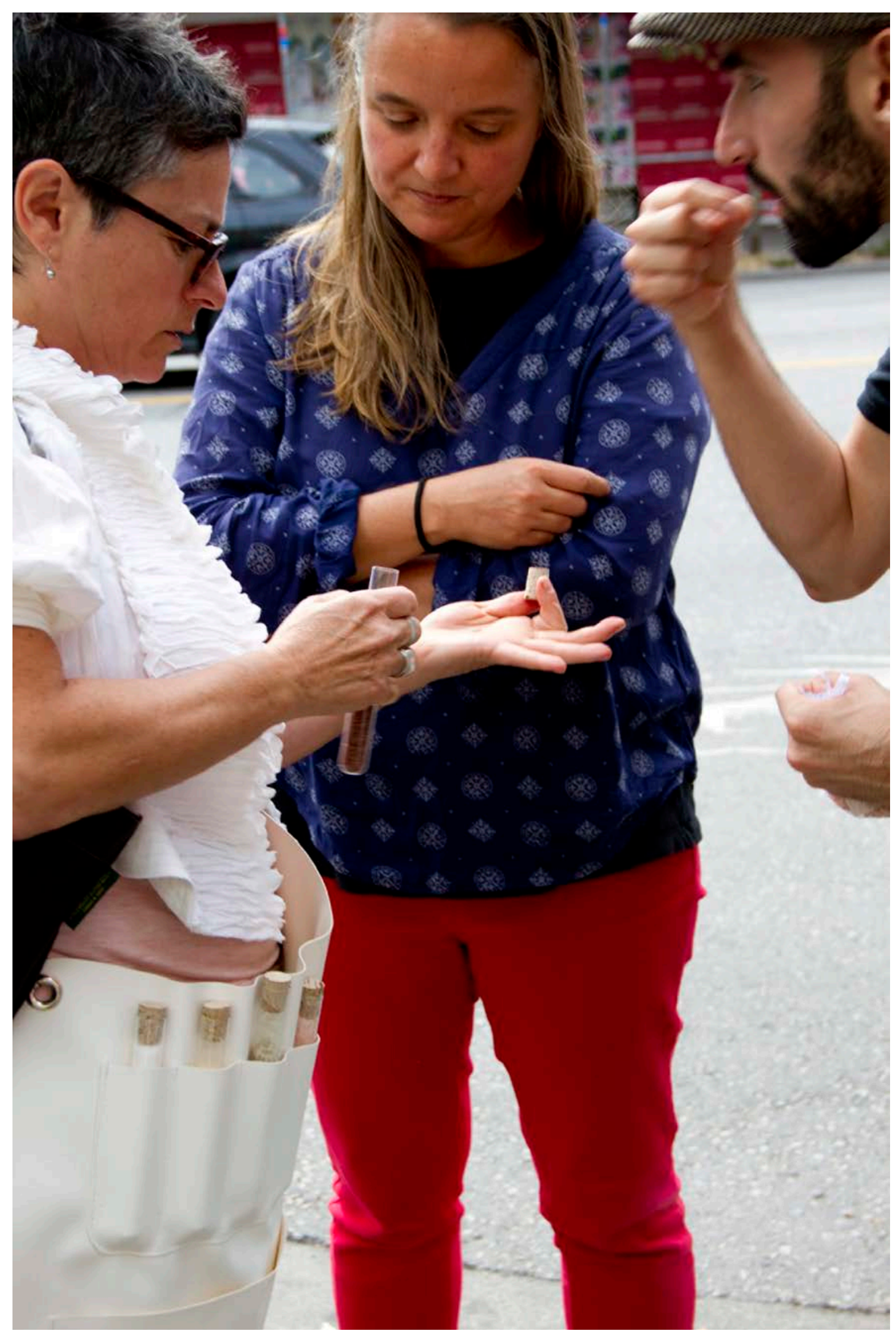

Figure 7: Randy Lee Cutler, 2013, SaltWalks: Vancouver, Access Art Gallery, Vancouver, BC. Photo credit: Dennis Ha. 


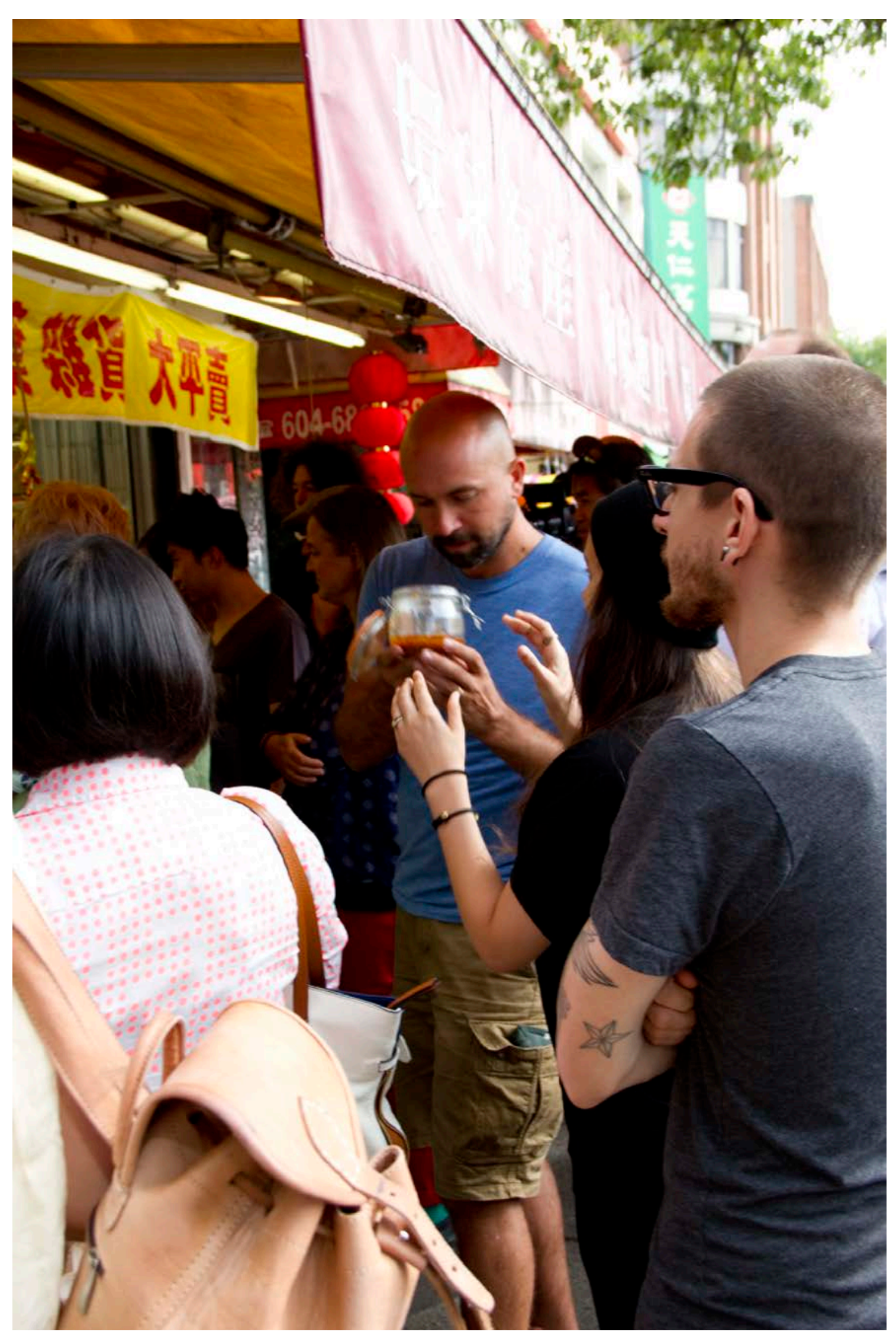

Figure 8: Randy Lee Cutler, 2013, SaltWalks: Vancouver, Access Art Gallery, Vancouver, BC. Photo credit: Dennis Ha. 


\section{References}

Haraway, D., (2015). Anthropocene, capitalocene, Chthulhucene: Donna Haraway in conversation with Martha Kenney. In H. Davis and E. Turpin (Eds.). Art in the anthropocene: Encounters among aesthetics, politics, environment and epistemology (pp. 255270). London, UK: Open Humanities Press.

Springgay, S., Truman, S. (2018). Walking methodologies in a more-than-human world: WalkingLab, New York, NY: Routledge.

Randy Lee Cutler is an artist, writer, and educator investigating the emergence of new cultural forms. At the intersections of gender, art, science, and technology, her practice takes up themes of hybrid research, materiality and sustenance. Randy is a professor at Emily Carr University in the Audain Faculty of Art, on the unceded Coast Salish territories also known as Vancouver, Canada. 